\title{
Editorial
}

\section{Special Issue on Social Work in the Time of COVID-19}

by

Mary Dallas Allen

Professor of Social Work, PhD

University of Alaska Anchorage

United States

E-mail: mdallen7@alaska.edu

Debbie Gonzalez

Lecturer of Social Work, DSW

Humboldt State University

United States

E-mail: debbie.gonzalez@humboldt.edu

Lennart Sauer

Lecturer of Social Work, PHD

Umeå University

Sweden

E-mail: lennart.sauer@umu.se

DOI: https://doi.org/10.31265/jcsw.v16.i2.490

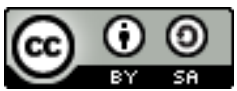

This work is licensed under a Creative Commons Attribution-ShareAlike 4.0 International License. 


\section{Introduction}

We are pleased to present the first of two editions of the special issue on social work in the time of COVID-19.

The University of the Arctic (UArctic) Thematic Network on Social Work (TNSWK) is a collaboration between international social work educators across eight different Arctic nations with a long-standing international relationship focused on northern voices and issues in social work education, practice and policy. The UArctic TNSWK is committed to promoting research and educational opportunities related to emerging social work issues in the Arctic. When the first wave of the COVID-19 pandemic resulted in worldwide travel bans and lockdowns, the TNSWK had been planning a summer 2020 in-person research meeting in Frankfurt, Germany. Since we were unable to meet to plan the Network's next steps in research, we shifted our focus to understanding the research scholarship on the impact of COVID-19 on social work education and practice, and decided to pursue a special journal issue on the topic. The Network has partnered with the Journal of Comparative Social Work for many years, so the TNSWK and the Journal of Comparative Social Work agreed to collaborate on the special issue on social work in the time of COVID-19. Due to the overwhelming response from the call for papers, the special issue will be published in two issues.

The special issue on social work in the time of COVID-19 examines the experience of social work practice and education during the first wave of the COVID-19 pandemic. The call for papers for the special issue was released at the beginning of September 2020, and abstracts were due on October 30, 2020. We received over 50 abstracts in response to the call for papers, which speaks to the immediate response of social work researchers across the world to generate knowledge about how the COVID-19 pandemic has affected social work education, practice and vulnerable populations. This two-edition special issue will be unique in that it provides a snapshot of the early days of the pandemic, when there were many more questions than answers.

The two editions of the special issue will highlight aspects of social work and social work education in the time of COVID-19. People across the globe are affected in different ways by the prevalent COVID-19 pandemic, and it has been a challenge for 
social work research, education and practice. This special issue provides the opportunity to share some of the experiences made through research on social work and social work education related to COVID-19. The articles in the special issues deal with social work practice during COVID-19, a comparative analysis between different countries, cultures and contexts related to social work and COVID-19, social work education and field (practical) education in the time of Covid-19, as well as the consequences of COVID-19 for different target groups and for social workers.

When communities began locking down and issuing social distancing and isolation protocols at the start of the COVID-19 pandemic, social workers, social service organizations and schools of social work experienced unprecedented service delivery challenges. And as social workers and social work educators scrambled to support their clients and organizations, they themselves experienced pandemic-related stress and worry at home in their personal lives. It was a time of incredible uncertainty, which was compounded by a loss of connection and community. The articles in this special issue explore these experiences across three themes: impacts on clients, impacts on social work education and consequences for social work practice and the profession.

\section{Impacts on Clients}

The theme impacts on clients highlights that the welfare state and social workers were put under pressure during the pandemic. Within this theme, the articles provide an analysis of the consequences of the Covid-19 pandemic for different target groups and social workers' concerns for their clients. When it comes to consequences for clients, the articles in this theme focus on the ethical challenges social workers deal with, the consequences of the shutdown of service and the concern that client groups in the most need and the most marginalized are the most affected. During the COVID-19 pandemic, many vulnerable groups experienced increased loneliness and mental health challenges, increased difficulty with managing social challenges and limited access to the Internet and digital tools. One of the areas within this theme is the pandemic's impact on children's well-being with a loss of physical contact, stress, sleep deprivation and sadness among children. When it comes to social workers' concerns for their clients, social workers experienced multiple work-related challenges, including difficulties with communication, worries about the clients' life 
situation, that the most vulnerable are the most affected and the difficulties of delivering social work services electronically.

The article, Social workers' perceptions on clients' service needs and social work practices during the COVID-19 pandemic, authored by Jokela, Kivipelto and Hiilamo, focuses on circumstances in Finland. The article describes the social workers' perceptions on how well social services were able to respond to clients' needs during the pandemic, and what practices in adult social work were used and developed during the outbreak of the pandemic. The data was generated from a national survey data, and the findings show that social workers could respond to clients' concrete problems, while they could not adequately respond to problems that were more complex. The findings also illustrate that social workers found that clients with more complex problems were the most affected by the COVID-19 pandemic.

The article, Downward spirals to vulnerability: Social workers' concerns about their clients during the first wave of the COVID-19 pandemic by Put, Bastaits, Pasteels and Massart focuses on social workers' and social service professionals' in Belgium (Flanders and the Brussels region) concerns about clients. The results are based on a large-scale online survey, with the results showing that social workers are concerned about their client's isolation, shrinking social networks and/or (increasing) Ioneliness. They also have a fear that the pandemic situation will lead to an aggravation of mental or psychosocial problems. Further concerns are related to increasing financial problems, and expectations that the clients will have increased family or relationship problems.

During the first wave of COVID-19, social workers were not only concerned with the Ioneliness of isolation, but also with the dangers of not being seen in the community, especially for the most vulnerable. In Business as usual in unusual times: An explorative study of Norwegian child welfare, Krane examines how Norwegian child welfare workers developed new assessments to determine when a case would be handled with a face-to face or virtual response. The study is a qualitative study of the difficulties, struggles, adaptations and strengths of 36 Norwegian child welfare social workers and nine child welfare leaders. The qualitative study explores the difficulty of addressing confidentiality and safety when conducting virtual interviews, in addition to the difficulties the workers experienced conducting child welfare work from their own 
homes isolated from their peers. The study highlights child welfare social workers concern for children whose families were involved with the child welfare system during the pandemic. The lockdowns and infection prevention measures resulted in many children losing an important connection to the community that helped keep them safe. The lockdown also placed child welfare workers in a precarious predicament in attempting to find a balance between providing help, support and assessment to their clients while simultaneously safeguarding their own families from the risk of the virus. Social work is relational work; the pandemic disrupted the connection between workers, clients and peers, and so engendered innovative approaches to effective social work practice.

\section{Social Work Education}

In March 2020, universities across the world responded to COVID-19 community lockdowns by closing campuses and moving courses online. For social work education, this had significant impacts on students, instructors and field education supervisors and instructors. In Key competencies in social work through field placement: Teaching and learning in Ukraine in the time of COVID-19, Gusak and Volhyna examine the effects of COVID-19 on field education in the Ukraine, using interviews with field supervisors, a student survey and student final self-assessment of competencies. The study describes the challenges that students experienced when the dormitories closed and they returned home to complete their college courses online. Students struggled with accessing online education due to lack of Internet access, limited digital skills and lack of access to computers. Moreover, students experienced multiple stressors, including isolation and a loss of support from close friends. COVID-19 has also impacted social work education instructors/lecturers who experienced multiple changes at once, which required them to negotiate with field agencies, identify new online field placements, learn new digital tools and develop new procedures for signing documents and meeting with field agencies. This study found that when classes were moved online at the beginning of the lockdown, the university was not prepared, as there were not enough licensed accounts, some lecturers had never used web conferencing and some lecturers were not trained in the learning management system. 
Jessica Ossais and colleagues explore how the pandemic affected field placements in Canadian social work field education during a global pandemic: A comparison of student and field instructor perspectives. For this study, the authors collected online surveys of field instructors and BSW and MSW students attending CASWE-ACFTS accredited schools of social work in Canada. The article examines the impact of COVID-19 on students' mental health and their experience of field education. Students reported that COVID-19 and social isolation negatively impacted their mental health, and that they had difficulty maintaining a work and home life balance during their practicum. Regarding their experience with field education, students reported a reduction in practicum hours, placements ending early, placements moving online, reduced learning goals and expectations, as well as changes in field placements. Students also reported diminished learning experiences due to the lack of a face-to-face practice. Students were concerned that ending their practicum early reduced their ability to complete practicum activities, limited their closure with clients and staff, and might have had a negative impact on clients. Most students in this study reported access to technology to complete their practicum, and many students did not perceive technology to be a barrier. This article also describes field instructors' experience of COVID-19. Field instructors also reported that COVID-19 impacted their mental health and wellness, and they reported multiple impacts on field education, including a partial or complete transition to online field education, a reduction in field education hours and their practicum terminating early. In this study, field instructors reported that they received adequate training on technology, and were satisfied with the support and resources provided by the institution. Lastly, Ossais et al. report that students and field instructors had different levels of satisfaction with supervision, as field instructors reported more satisfaction with the level of supervision than students.

\section{Consequences for Practice and Profession}

During the first wave of the COVID-19 pandemic, social workers and social service organizations were not prepared for the impact of infection prevention and control measures on social work practice, or for the health and mental health effects of a global pandemic. As agencies closed during lockdowns, many social service organizations transitioned to distance-delivered telehealth services, and workers began providing services from home. Consequently, social workers developed new 
insights on the provision of remote social work services, managing online meetings and balancing work and family responsibilities. However, the pandemic also highlighted inequalities in access to technology, the Internet and the digital infrastructure. In their mixed methods study of Canadian child welfare workers, Baker, Berens, Williams, Bruer, Evans and Price describe the impact of the first and second waves of COVID-19 on rural and urban social workers' perceptions of safety, stress, worry and organizational support. They report that rural and urban child welfare social workers made similar modifications to service delivery through remote delivery, physical distancing and personal protective equipment (PPE) use. In addition, rural social workers began providing services outdoors in wave two. The authors report that in the first wave of the pandemic, rural social workers reported more stress, worry and a need for mental health services, while in phase two urban social workers reported an increase in stress and a need for mental health services. Rural social workers also experienced specific challenges related to limited access to technology, the Internet and infrastructure. This paper emphasizes the importance of supporting social workers' overall well-being to reduce burnout.

In the article by Aasback and Røkkum, Domesticating Technology in Pandemic Social Work, the authors explore how social workers in Norway adapted the use of digital technology to deliver services and support based on relationships, community building and the values of social work within a digital medium. The article explores a common theme of how technology influenced the delivery of social work practices and expanded social worker's digital tool box in the first wave of the pandemic. Access to technology and knowledge of how to use technology emerged as an important skill to sustain professional relationships and a sense of community in the early stages of the lockdowns. The pandemic created a parallel struggle experienced by social work professionals and clients, but highlighting the importance of continued contact for vulnerable clients. The authors' conceptual framework of domestication theory provides a perspective on the role social workers and clients had in "tinkering" with the integration of technology into social work practice.

The articles on field education in Canada and Ukraine by Ossais et al. and Gusak and Volhyna also provide insight into the future of social work education and field education. The article by Gusak and Volhyna describes the innovations in field 
education in the Ukraine that occurred during the pandemic, such as using online applications to allow courses to be more flexible, improved communication between instructors, field supervisors and students, and the creation of creative new field education partnerships. In their study on field education in Canada, Ossais et al. describe that students and field instructors reported learning new skills during the pandemic. Students reported improvement in virtual skills, self-motivation, selfdirected study, research skills, disaster skills and community understanding, whereas field instructors reported learning new skills from students and from using technology to connect with clients and students. These findings provide insight into skills that social workers need during pandemic times. The article by Ossais et al. reports concerns from students and field instructors that students may not have experienced sufficient learning in the field to have developed the skills needed post-graduation. Students felt they did not have enough learning to prepare for professional practice, and did not get enough experience for future jobs. Students also expressed concern about being able to pay tuition during the pandemic and being able to pay off loans after graduation, which has implications for the future of social work practitioners.

\section{Conclusion}

The ongoing COVID-19 pandemic is still impacting the world, and we will be working to understand the impacts of the pandemic on the field of social work for many years to come. Volume 1 of the special edition provides a snapshot of how social work educators and practitioners adapted to the first wave of the pandemic from five different Arctic nations, including Finland, Belgium, Norway, Ukraine and Canada. Infection prevention and control measures, such as lockdowns, has had an impact on face-to-face services delivery, peer support and social work education, though we learned how social workers adapted with innovation, creativity and flexibility across the North. Social workers and social work educators remained committed to the wellbeing of their clients and students, and dedicated to furthering the goals of best social work practice in the face of a global health crisis. The impact of the COVID- 19 pandemic had a tremendous impact on social work education and social work practice, particularly in the application of technology in education and practice. This special issue highlights the importance of understanding the use of technology for social work education and practice. 